\title{
MODELLING SOIL-FIBRE COMPOSITE BEHAVIOUR USING A MICROMECHANICAL APPROACH
}

\author{
Thomas A. Bower ${ }^{1}$, Anthony D. Jefferson ${ }^{2}$, Peter J. Cleall ${ }^{2}$ and Paul Lyons ${ }^{3}$ \\ ${ }^{1}$ School of Engineering, Cardiff University \\ Queen's Buildings, The Parade, Cardiff, CF24 3AA, UK \\ e-mail: BowerTA@ cardiff.ac.uk \\ ${ }^{2}$ School of Engineering, Cardiff Univerisity \\ Queen's Buildings, The Parade, Cardiff, CF24 3AA, UK \\ e-mail: $\{$ JeffersonAD,Cleall $\} @$ cardiff.ac.uk \\ ${ }^{3}$ LUSAS \\ Forge House, 66 High Street, Kingston Upon Thames, Surrey, KT1 1HN, UK \\ e-mail: paul.lyons@lusas.com
}

Keywords: composite, soil, fibre, constitutive model, shear lag, debonding

\begin{abstract}
Experimental data has shown that the addition of manufactured fibres to a soil can significantly increase its strength. The mechanism of strength increase is due to the frictional interaction between the soil particles and each fibre. This has a global effect of increasing the shear strength of the soil. This paper presents the initial development of a micro-mechanics based soil-fibre model. This model considers the micromechanical soil-fibre interaction and in particular utilises the shear lag model which is extended to include fibre de-bonding. The effects of a single fibre are then homogenised using a numerical spherical integration technique to take into account the distribution of fibre orientations. The fibre model is used in conjunction with a hardening soil plasticity model. The proposed soil-fibre model is then compared against experimental data for triaxial compression of fibres mixed with a well graded sand and different fibre concentrations. The volumetric response shows the correct trend with different fibre contents however the actual values are somewhat inaccurate, The shear stress response is very well captured. It is concluded that the results could be improved by addressing the known limitations in the base soil model and the fibre model. Future work will involve the improvement of both.
\end{abstract}




\section{INTRODUCTION}

The requirements for engineering soils are becoming more and more demanding, with larger structures being built and less choice in where to put them, engineers have fewer options with site selection and soils often need to be improved. This can be achieved using methods such as vibro-compaction and drop weight compaction to increase the soil density, or by importing stronger soils. Geosynthetics is another option to improve soil strength which can be used in conjunction with other methods. These include geogrids, geomats, geomembranes and fibre reinforcement. One advantage of using fibre reinforced soils over planar grids / mats is that they do not introduce any particular planes of weakness.

A variety of fibre materials are available for soil reinforcement. Possibly the original application of fibre reinforcement is the use of plant roots which have been used historically for the stabilisation of slopes and embankments. More recently, investigations have been undertaken into the behaviour of manufactured fibres in soils. Such studies have looked at fibres constructed from plastics such as polypropylene [5, 9, 11] and polyamide [15], or natural fibres such as coir [13] and oil palm [1]. Figure 1 depicts the crimped polypropylene fibres used in this study.

The primary mechanism for strength increase is due to the interfacial friction between the soil particles and the fibre: isotropic pressure compacts the composite material, causing soil particles to partially embed in the fibre. The presence of the fibre resists shear displacements hence increasing the shear strength of the soil.

Fibres used for reinforcing soils must be significantly stronger than the soil mass. It would be futile adding fibres which would break before soil failure. Manufactured fibre materials such as polypropylene and polyethylene generally do not reach the yield stress within the strain range observed in soils; also axial fibre strains dominate radial fibre strains by far; thus fibres are typically considered as one-dimensional axially loaded elastic elements in fibre models.

Several soil-fibre models have been developed in recent years. Namely, Maher and Gray [12] created a micro-mechanical soil-fibre interaction model whereby fibres crossing potential shear planes increased the shear strength across that plane. The effects on a single direction were then homogenised using a spherical integration technique. Michalowski and Čermák [15] developed a failure criterion for fibre reinforced soils whereby a Mohr-Coulomb type cone model was expanded due to the presence of fibres. Diambra et al. [8] used the rule of mixtures to create an equivalent fibre stiffness matrix which was superimposed onto the soil stiffness matrix. A relationship for fibre debonding was included in this model and later improved by Diambra and Ibraim [10].

\section{FIBRE MODEL}

The first step in modelling soil containing randomly distributed fibres is to analyse the effect a single fibre has on the soil surrounding it; an idealised schematic of the soil and fibre is shown in Figure 2. By taking an infinitesimal slice of fibre / soil, and balancing the forces due to the applied matrix strain $\varepsilon_{m a}$ leads to the well known shear lag equation [7].

$$
\frac{\mathrm{d} \sigma_{f a}}{\mathrm{~d} x}=\frac{-2 \tau}{r_{f}}
$$

where $\sigma_{f a}$ is the fibre tensile stress, $x$ is the distance from the fibre centre, $\tau$ is the interface shear stress, $r_{f}$ is the fibre radius. Through manipulation of Eq. 1. using stress/strain relationships in the fibre and soil, the slip between the matrix and fibre at any point along the fibre is found to 




Figure 1: Polypropylene Loksand fibres used in experimental study [6]

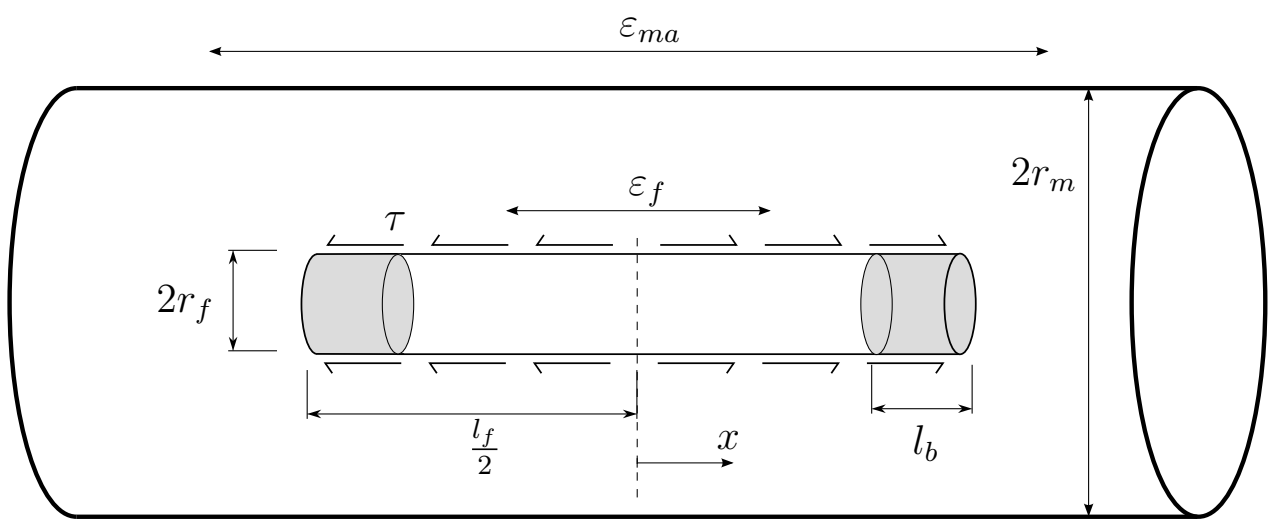

Figure 2: Single fibre and surrounding matrix material, showing debonding regions

be

$$
S(x)=\frac{\varepsilon_{m a}}{\beta \cosh \left(\beta l_{f} / 2\right)} \sinh (\beta x)
$$

where $\beta$ is a constant relating various properties of the soil, the fibre and the interface. As the matrix strain $\varepsilon_{m a}$ increases, the interface shear stress also increases, up to a limit $\tau_{b}$. With further loading, the limiting shear stress will expand towards the fibre centre. The length of this debonded region is given by $l_{b}$ which is calculated using the applied matrix strain.

$$
S(x)= \begin{cases}\frac{\tau_{b}}{k_{s}} \frac{\sinh (\beta x)}{\sinh \left(\beta\left(l_{f} / 2-l_{b}\right)\right)} & \text { if } 0<x \leq l_{f} / 2-l_{b} \\ \frac{\tau_{b}}{k_{s}} & \text { if } l_{f} / 2-l_{b}<x \leq l_{f} / 2\end{cases}
$$

The fibre tensile stress is found by integrating the slip relations from the fibre centre to the desired position. This applies to both the bonded case 2 and the debonding case 3 . The axial stress and shear stress distribution functions are visualised in Figure 3 . 


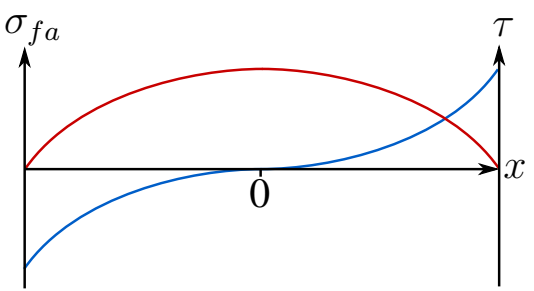

(a) Fully bonded

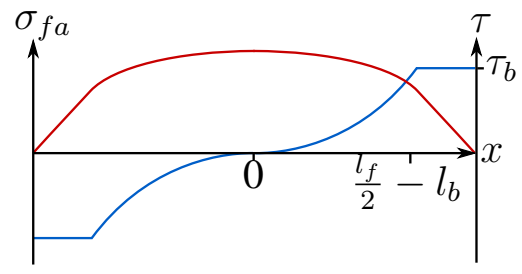

(b) Partially debonded

Figure 3: Tensile fibre stress $\sigma_{f a}$ (red) and interface shear stress $\tau$ (blue) distributions for bonded and partially debonded fibre along the length of the fibre [4]

$$
\sigma_{f a}=\frac{-2 k_{s}}{r_{f}} \int_{0}^{x} S(x) d x
$$

\section{SPHERICAL INTEGRATION}

To translate individual fibre stresses to global stresses suitable for use in finite element analysis, the behaviour of the fibres must be homogenised. Another important factor at this stage is the distribution of fibre orientation. The sample preparation method has a significant impact on the fibre orientations [11, 17]. In a triaxial compression test, the vertical fibres have little impact on the results, whereas fibres on the horizontal plane resist the tensile strains and affect the results significantly. The orientation distribution function used in this study [14] is given in Eq. (5) with the parameters $A=0, B=0.57, n=7$ based on results reported by Wang [17].

$$
\rho_{\theta}=A+B\left|\sin ^{n} \theta\right|
$$

The distribution is then used in a numerical spherical integration method [2]. This approach is similar in form to Gauss-Legendre integration used in finite element formulations, summating the fibre effects over a number of sampling directions and applying sampling weights to each direction.

$$
\sigma_{f}=v_{f} \sum_{i_{d}=1}^{n_{d}} \rho\left(\theta_{i d}\right) \cdot \chi\left(\theta_{i d}\right)^{T} \int_{0}^{l_{f} / 2} \frac{2}{l_{f}} \sigma_{f a}\left(x, \varepsilon_{m a}\right) \mathrm{d} x \cdot w_{i d}
$$

The integral term in Equation (6) takes into account the different positions of the fibres. $n_{d}$ is the number of sampling directions, $\sigma_{f a}$ is the fibre axial stress for either fully or partially bonded fibres and $w_{i d}$ is the current sample weight. $\chi\left(\theta_{i d}\right)$ forms a direction cosine vector for each sampling direction. This vector translates the fibre stresses into the global stress space (Cartesian, axisymmetric etc.). The same vector is also used to translate the global strains into the applied axial matrix strain $\varepsilon_{m a}$.

\section{THE HARDENING SOIL MODEL}

The hardening soil model, initially developed by Schanz [16] and later improved by Benz [3], is a constitutive soil model capable of capturing non-linear behaviour exhibited by many soils including stress dependent stiffness, dilatancy and hardening from initial loading. The model performs well for simulating both laboratory tests and more complex boundary value 
problems. The HS model relies on a set of yield surfaces which define the boundary of elastic and plastic strains. The shear surface (7) is a curved cone in principal stress space (see [16]).

$$
f_{s}=\frac{q_{a}}{E_{50}} \frac{q}{q_{a}-q}-\frac{2 q}{E_{u r}}-\gamma_{p}
$$

where $q_{a}$ is the failure shear stress (governed by a Mohr-Coulomb criterion), $E_{50}$ and $E_{u r}$ are the current secant and unload-reload stiffnesses, $q$ is the shear stress and $\gamma_{p}$ is the current value of the plastic shear strain and controls the size of the shear surface. This is a non-associated surface and the plastic potential in shearing is the Drucker-Prager cone with the dilatancy angle $\psi$ controlling the cone steepness. The cap surface takes the form of an ellipse in $\mathrm{p}-\mathrm{q}$ stress space.

$$
f_{c}=p^{2}+\frac{q^{2}}{\alpha^{2}}-p_{p}^{2}
$$

where $p$ is the mean stress, $\alpha$ is a parameter which controls the cap steepness and $p_{p}$ is the equivalent pre-consolidation stress and controls the size of the cap surface. The cap surface uses an associated flow rule therefore the plastic potential function is equal to the yield function. During drained triaxial testing, both surfaces are active, therefore a double hardening closest point projection method is employed.

\section{RESULTS}

The predicted results were generated by a single point constitutive driver in Cartesian stress space using the fibre and soil properties listed in Table 1 and Table 2. An additional subset of soil properties were used in the hardening soil model, however they are not listed here. The triaxial test simulation was undertaken by supplying an initial isotropic stress of $0.3 \mathrm{~N} / \mathrm{mm}^{2}$, an axial strain was applied at a rate of $\Delta \varepsilon_{y}=0.6 \%$. Shear stress and volumetric strain curves are compared with experimental data [6] in Figure 4. A quadratic rate of convergence was observed throughout the simulations.

Table 1: Properties of the Loksand fibre

\begin{tabular}{|ccc|}
\hline $\begin{array}{c}\text { Young's modulus } \\
E_{f}\end{array}$ & $\begin{array}{c}\text { radius } \\
r_{f}\end{array}$ & $\begin{array}{c}\text { length } \\
l_{f}\end{array}$ \\
\hline $900 \mathrm{~N} / \mathrm{mm}^{2}$ & $0.044 \mathrm{~mm}$ & $35 \mathrm{~mm}$ \\
\hline
\end{tabular}

Table 2: Strength properties of the sand

\begin{tabular}{|ccccc|}
\hline Young's modulus & Poisson's ratio & Friction angle & Dilatancy angle & Cohesion \\
$E_{m}$ & $\nu$ & $\varphi$ & $\psi$ & $c$ \\
\hline $40.6 \mathrm{~N} / \mathrm{mm}^{2}$ & 0.3 & $35^{\circ}$ & $2^{\circ}$ & 0 \\
\hline
\end{tabular}

\section{DISCUSSION AND CONCLUSIONS}

The shearing response of an unreinforced soil starts with a high stiffness which reduces with increasing axial load until it reaches failure where no further shear stress can develop. For the reinforced soil, this failure limit is not reached (Figure 4a), and the shear stress is allowed to 




(a) Shear response

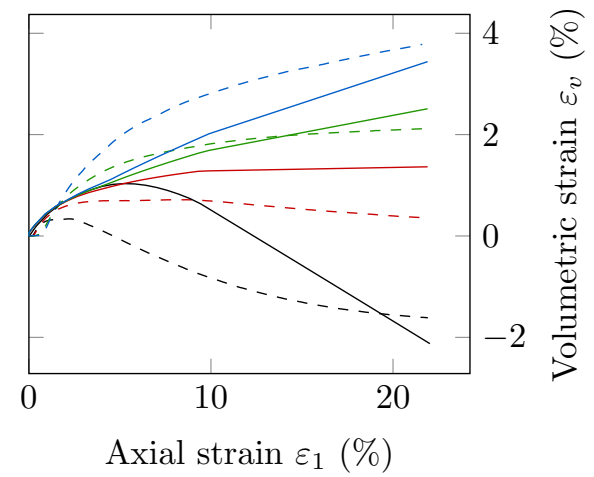

(b) Volumetric response

Figure 4: Simulated (solid) and experimental (dashed) drained triaxial results for fibre contents by weight of $0.0 \%$ (black), $0.3 \%$ (red), $0.6 \%$ (green) and $0.9 \%$ (blue); experimental results by Chatzopoulos [6]

increase with axial load indefinitely within the strain range considered. With increasing fibre content, this effect is increased. This behaviour is captured well in the soil-fibre model with only small differences in the shear response past $10 \%$ axial strain.

The initial response (lower than $10 \%$ axial strain) is somewhat mis-represented in shearing. The initial stiffness of the unreinforced sample is under-predicted; suggesting that there are some limitations in the base soil model. Also, the initial stiffness of the reinforced samples is shown to decrease with increasing fibre content; the fibre model does not reproduce this behaviour.

The unreinforced sand undergoes an initial compressive stage, then after 3\% axial strain (Figure 4b), begins to exhibit dilatancy. The predictions of this do show dilatancy but at a later strain of $6 \%$. This is most likely due to the simplified dilatancy rule used in the soil model. The experimental result also shows a dilatancy cut-off at $\varepsilon_{v}=-1.8 \%$, the model does not predict this.

As the fibre content is increased, the experimental results show a decrease in dilatancy. This trend is captured well however the values are less accurate than the shear response. The effects of the simplified dilatancy rule in the soil model may be dominating over the influence of the fibres, so it is difficult to comment on the accuracy of this element of the fibre model.

In summary, the predictions match the experimental results somewhat better than in previous work in this ongoing project [4]. In particular, the volumetric response is much better predicted. Further work will focus on the debonding behaviour of the fibres and the dilatant response of the soil.

\section{Acknowledgements}

The work presented here was supported by LUSAS / Finite Element Analysis ltd. and Innovate UK / TSB as part of KTP project number 9082. We would also like to thank Drake Extrusion Inc. for supplying the fibres used in the experimental testing.

\section{REFERENCES}

[1] F. Ahmad, F. Bateni, M. Azmi, Performance evaluation of silty sand reinforced with fibres. Geotextiles and Geomembranes, 28.1, 93-99, 2010. 
[2] Z.P. Bažant and B.H. Oh., Efficient numerical integration on the surface of a sphere. ZAMM - Journal of Applied Mathematics and Mechanics, 66.1, 37-49, 1986.

[3] T. Benz, Small-strain stiffness of soils and its numerical consequences. Univ. Stuttgart, Inst. f. Geotechnik, 2007.

[4] T.A. Bower, A.D. Jefferson, P.J. Cleall, P. Lyons A micro-mechanics based soil-fibre composite model for use with finite element analysis. submitted to Association of Computational Mechanics in Engineering, 2016.

[5] Y. Cai, B. Shi, C.W.W. Ng, C.S. Tang, Effect of polypropylene fibre and lime admixture on engineering properties of clayey soil. Engineering Geology, 87.3, 230-240, 2006.

[6] S. Chatzolpoulos, Soil reinforced with discrete polypropylene fibers. MSc Thesis, Cardiff University, 2015.

[7] H.L. Cox, The elasticity and strength of paper and other fibrous materials. British Journal of Applied Physics, 3.3, 72-79, 1952.

[8] A. Diambra, E. Ibraim, D. Muir Wood, A.R. Russell, Fibre-reinforced sands: experiments and modelling. Geotextiles and Geomembranes, 28.3, 238-250, 2010.

[9] A. Diambra, E. Ibraim, A.R. Russell, D. Muir Wood, Fibre reinforced sands: from experiments to modelling and beyond. International Journal for Numerical and Analytical Methods in Geomechanics, 37.15, 2427-2455, 2013.

[10] A. Diambra, E. Ibraim, Fibre reinforced sands: interaction at the fibre and grain scale. Géotechnique, 65.4, 296-308, 2015.

[11] E. Ibraim, A. Diambra, A.R. Russell, D. Muir Wood, Assessment of laboratory sample preparation for fibre reinforced sands. Geotextiles and Geomembranes, 34, 69-79, 2012.

[12] M.H. Maher, D.H. Gray, Static response of sands reinforced with randomly distributed fibers. Journal of Geotechnical Engineering, 116.11, 1661-1677, 1990.

[13] T. Maliakal, S. Thiyyakkandi, Influence of randomly distributed coir fibers on shear strength of clay. Geotechnical and Geological Engineering, 31.2, 425-433, 2013.

[14] R.L. Michalowski, J. C̆ermák, Strength anisotropy of fiber-reinforced sand. Computers and Geotechnics, 29.4, 279-299, 2002.

[15] R.L. Michalowski, J. Čermák, Triaxial compression of sand reinforced with fibers. Journal of Geotechnical and Geoenvironmental Engineering, 129.2, 125-136, 2003.

[16] T. Schanz, P.A. Vermeer, P.G. Bonnier, The hardening soil model: formulation and verification. Beyond 2000 in computational geotechnics, 281-296, 1999.

[17] Z. Wang, Fibre reinforced sand: from sample preparation to triaxial tests and orientation investigation. MSc Thesis, Cardiff University, 2015. 